AperTO - Archivio Istituzionale Open Access dell'Università di Torino

\title{
Atrial fibrillation in $\beta$-thalassemia Major Patients: Diagnosis, Management and Therapeutic Options
}

\section{This is the author's manuscript}

Original Citation:

Availability:

This version is available http://hdl.handle.net/2318/1678177

since 2019-06-01T16:42:57Z

Published version:

DOI:10.1080/03630269.2018.1488724

Terms of use:

Open Access

Anyone can freely access the full text of works made available as "Open Access". Works made available under a Creative Commons license can be used according to the terms and conditions of said license. Use of all other works requires consent of the right holder (author or publisher) if not exempted from copyright protection by the applicable law. 


\section{SHORT COMMUNICATION}

Atrial fibrillation in $\beta$-Thalassemia major patients: diagnosis, management and therapeutic options.

Umberto Barbero $^{\mathrm{a}, \mathrm{b}}$, Federico Fornari ${ }^{\mathrm{c}}$, Silvia Guarguagli ${ }^{\mathrm{a}}$, Carmen Maria Gagliotic, Filomena Longo $^{c}$, Baldassarre Doronzo ${ }^{b}$, Matteo Anselmino ${ }^{\mathrm{d}}$ and Antonio Piga ${ }^{\mathrm{c}}$

${ }^{a}$ Royal Brompton and Harefield Hospital, Imperial College London, London, UK; ${ }^{\mathrm{b} C a r d i o l o g y}$ Unit, "SS. ma Annunziata” Hospital, Savigliano, Italy; 'Dipartimento of Clinical and Biological Sciences, Turin University, San Luigi Gonzaga, Torino, Italy; ${ }^{\mathrm{d} C a r d i o l o g y}$ Unit, "Città della Salute e della Scienza” Hospital, University of Turin, Turin, Italy

Received 13 January 2018

Accepted 29 May 2018

Address correspondence to Dr. Umberto Barbero, Royal Brompton and Harefield Hospital, Imperial College London, Sydney Street, London SW3 6NP, UK. E-mail: ubarbero@unito.it. 


\section{SHORT COMMUNICATION}

Atrial fibrillation in $\beta$-Thalassemia major patients: diagnosis, management and therapeutic options.

Umberto Barbero $^{\mathrm{a}, \mathrm{b}}$, Federico Fornari ${ }^{\mathrm{c}}$, Silvia Guarguagli ${ }^{\mathrm{a}}$, Carmen Maria Gagliotic, Filomena Longo $^{c}$, Baldassarre Doronzo ${ }^{\mathrm{b}}$, Matteo Anselmino ${ }^{\mathrm{d}}$ and Antonio Piga ${ }^{\mathrm{c}}$

${ }^{a}$ Royal Brompton and Harefield Hospital, Imperial College London, London, UK; ${ }^{\text {bCardiology }}$ Unit, "SS. ma Annunziata” Hospital, Savigliano, Italy; cDipartimento of Clinical and Biological Sciences, Turin University, San Luigi Gonzaga, Torino, Italy; dCardiology Unit, "Città della Salute e della Scienza” Hospital, University of Turin, Turin, Italy

\section{Abstract}

The prevalence of atrial fibrillation (AF) in $\beta$-thalassemia major ( $\beta$-TM) patients has increased in the last few years, reaching up to $33.0 \%$. Several factors may drive this value to even more in the next years. We summarized the main challenges in the management and therapy of AF in this very specific group of patients.

KEYWORD Arrhythmia; atrial fibrillation (AF); $\beta$-thalassemia ( $\beta$-thal); iron overload

$\beta$-Thalassemia major ( $\beta$-TM) is a hereditary hemoglobin $(\mathrm{Hb})$ disorder caused by reduced synthesis of $\beta$-globin chain and resulting in a chronic hemolytic anemia that typically requires life-long transfusion therapy. If left untreated, this could result in growth retardation, bone marrow expansion, extramedullary hematopoiesis, splenomegaly, greater intestinal iron absorption, hypercoagulability and higher susceptibility to infections till death occurs [1-3].

Although traditionally prevalent in the Mediterranean Basin, Middle East, North India, and Southeast Asia, migration of those populations to North America and Western Europe has rendered $\beta$-thalassemia ( $\beta$-thal) a global health problem. According to the Thalassemia International Federation, (Nicosia, Cyprus), about 200,000 people are affected by $\beta$-TM and registered as receiving regular treatment around the world [4]. The highest carrier frequency is reported in Cyprus (14.0\%), Sardinia (10.3\%) and Southeast Asia [5].

Thanks to transfusions and iron chelation, the prognosis of $\beta$-TM has dramatically improved, and recent studies have shown that the $80.0 \%$ of patients in countries with modern therapies available, survive to over 40 years of age [6,7]. A further improvement of survival for these patients is to be expected from an earlier commencement of chelation therapy and, in the near future, with the introduction of new drugs such as Luspatercept and genetic therapy.

Despite the improvements in both therapies and follow-up modalities, cardiac complications represent the primary cause of mortality and one of the major causes of morbidity in those patients [8-10]. The first reason of cardiac damage is iron overload, due mainly to repetitive blood transfusions, as well as hemolysis and increased intestinal absorption. The increase of plasma iron turnover results in saturation of the iron storage and transport proteins and the 
appearance of a free plasma iron pool. The iron-mediated oxidative stress can damage cardiomyocytes causing lipid peroxidation, mitochondrial injury, membrane disruption, and contractile dysfunction. Advanced iron overload, along with inflammation and immunogenetic factors, leads to cardiac chamber dilation and focal myocardial degeneration and fibrosis. Involvement of the conduction system is less severe and inhomogeneous. Sometimes pericardial and pulmonary vascular abnormalities are also present $[1,9]$.

Heart failure usually develops in patients with non optimal chelation therapy and multiendocrinopathies. The incidence of iron overload cardiomyopathy ranges from 11.4 to $15.1 \%$ in $\beta$-TM patients [7,8]. Restrictive cardiomyopathy usually occurs first followed by dilatation, in accordance with diastolic dysfunction, which normally precedes systolic dysfunction and overt heart failure. From the earliest reporting of the cardiovascular complications of thalassemia, arrhythmias were noted to feature prominently [11]. Findings in the early stage are usually incidental and include bradycardia, repolarization changes, infrequent premature atrial or ventricular contractions and first-degree atrioventricular blocks. In the late stage, atrial and ventricular tachycardia and second-degree or complete heart block are common findings [8].

In atrial tachycardia, the prevalence of atrial fibrillation (AF) is high and, due to longer survival of thalassemic patients, may increase in the future with important clinical implications. While iron-induced cardiomyopathy is slowly progressive and it usually takes several decades to manifest, AF can already be present at the earlier stage of isolated myocardial siderosis [8]. The prevalence of AF in adult $\beta$-TM patients ranges from 10.0 to $33.8 \%$ in the literature, also depending on the study methods used to detect AF [12-14]. Atrial fibrillation is frequently badly tolerated and is a concern for the patients and a clinical problem for the physician.

Furthermore, which is the possible explanation for this higher prevalence of AF in $\beta$-TM patients? That brings us back to the concept of Coumel's triangle. According to Coumel, 'there are always three main ingredients required for the production of a clinical arrhythmia: the arrhythmogenic substrate, the trigger factor and the modulation factors of which the most common is the autonomic nervous system dysfunction' [15]. All the elements are present in $\beta$ TM patients. The arrhythmogenic substrate is represented by the iron overload and left atrium dilatation.

Early pathological examination of hearts affected by iron overload emphasized the patchiness of iron overload [11] with variable iron content present also in the conducting tissue. Iron-loaded myocytes show abnormal action potentials with decreased overshoot and shortened action potential duration. These features are likely to occur in a patchy distribution at a microscopic level and may constitute the electrical substrate for arrhythmogenesis [16]. In a prospective series of patients undergoing cardiac magnetic resonance (CMR) scans to detect iron-induced fibrosis [17], about $14.0 \%$ of patients with severe iron overload (i.e. T2* $<6 \mathrm{~ms}$ ) experienced an arrhythmia within 1 year from the CMR scan, and most of these arrhythmias were AF ( $79.6 \%$ of patients). Nevertheless, arrhythmias may also occur in patients with a normal myocardial T2*. Possible explanations include the existence of atrial iron loading separate from the ventricle, the persistence of atrial iron loading despite ventricular clearance, myocarditis, and the longstanding pro arrhythmic atrial effects of volume loading and high cardiac output caused by chronic anemia [18]. 
Left atrium dilatation is more frequent in $\beta$-TM patients than in healthy controls and it is considered an early feature of cardiac remodeling in $\beta$-TM [19]. P-wave dispersion is a noninvasive indicator of intra-atrial conduction heterogeneity associated with atrial remodeling. It is the substrate for re-entry, which is one of the pathophysiological mechanisms of AF. In the study by Russo et al. [20], P-wave dispersion (defined as the difference between the maximum $\mathrm{P}$-wave duration and the minimum P-wave duration in the same patient) was significantly increased in $\beta$-TM patients with AF compared to those without and to normal controls, and correlate with myocardial iron deposit assessed by CMR-T2* star imaging. This was confirmed in a second study by the same group [12] using external continuous loop recording. Based on these reports, an abnormal P-wave dispersion $\geq 35.5 \mathrm{~ms}$ and maximum P-wave duration $\geq 111 \mathrm{~ms}$, can predict AF onset in $\beta$-TM patients with conserved systolic and diastolic function [12]. However, AF itself is only one of multiple factors as seen in patients affected by $\beta$-thal intermedia ( $\beta$-TI) who have less cardiac arrhythmia compared to $\beta$-TM patients, despite larger chamber volumes. The little cardiac iron found in $\beta$-TI patients implies a possible critical role of the iron toxicity and the vegetative neural impairment [21].

As regards the trigger factors leading to the occurrence of AF, ventricular extrasystoles, as well as infrequent premature atrial or ventricular contractions and first-degree atrioventricular blocks are common findings in $\beta$-TM patients; furthermore, these patterns are detectable starting from very early stages of cardiac iron overload [8].

The third factor of Coumel's triangle is modulation factors and, among others, the most important is the nervous system. The $\beta$-TM patients present with autonomous nervous system dysfunction contributing to arrhythmogenesis $[14,18]$. Patients with $\beta$-TM in fact have been shown to have a lower heart rate variability (HRV) compared to normal subjects [22-24]. Moreover, the decline in HRV precedes the clinical signs of cardiomyopathy. As suggested by Yetimakman et al. [25], in the absence of systolic or diastolic dysfunction, HRV parameters can be used to detect early cardiac autonomic involvement, which together with atrial dilatation creates a substrate that favors the development of arrhythmias, especially AF. Despite the small number of patients included in all the studies we quoted, they all seem to come to the same conclusion.

The absence of studies involving large populations with the disease is a limiting factor for a better understanding of the pathology and its consequences. There is, for example, a wide range in the published literature regarding the prevalence of AF in $\beta$-TM patients.

In the first studies, its prevalence was around $10.0 \%[7,8]$ but that has reached up to $33.8 \%$ in recent times $[11,13,26]$. This increase should alarm $\beta$-TM specialists. Atrial fibrillation, in fact, is associated with morbidity and increased risk of mortality; stroke is a frequent complication of $\mathrm{AF}$, and its risk is associated with several clinical factors summarized by the CHA2DS2-VASc scoring system [27]. The growing age of thalassemic population likely contributes to the increasing prevalence of AF and, considering the longer survival due to modern treatments, the occurrence will probably increase further in the near future. A potential epidemic of AF could involve the $\beta$-TM population and, considering its potential detrimental damages, physicists should be aware of how to face it. Effective treatment starts with early recognition and active 
screening should be suggested to patients, even when cardiac function still appears normal, as shown in a recent long-term prospective observational study [28] that revealed an overall AF prevalence of $20.9 \%$ in a large $\beta$-TM-population with a normal cardiac function undergoing long-period, external loop recorder (ELR) monitoring every 6 months during their 5 -year followup. We summarize below what appears a reasonable outline for the management of the antiarrhythmic and anticoagulant therapy in this very specific group of patients.

1) Rate control vs. rhythm control: as patients with $\beta$-TM are usually quite young even when developing AF, which is the biggest clinical difference in respect to normal AF management, rhythm control should always be pursued as long as it is possible. Due to the fact that AF tends to be highly symptomatic in these anemic young patients, who often already have a hyperdynamic circulatory setting, rate control is often necessary. Due to the lack of evidence in the field, both $\beta$-blockers and calcium antagonist (diltiazem, verapamil) may be suggested in the presence of normal ejection fraction. Caution is needed in patients whose echo shows early tissue Doppler abnormalities or who have a history of previous heart failure; in these patients, low doses titration is advised. In patients with low $\mathrm{T} 2 *$ values or impaired ejection fraction, digoxin might instead be used.

In an acute iron overload state or every time the iron plasmatic level is not adequately controlled, the mainstay of the AF treatment as well as of the whole spectrum of thalassemic cardiomyopathy is chelation therapy. This means that after an initial period of some months for rate control, AF management could be switched to rhythm control. This should be addressed especially because the thrombotic risk is less predictable in such patients, on one hand, because of the different age scale (with unknown threshold for risk that are surely different from the score of a patient who is 64 years old) and on the other hand, because the same thalassemic patients who could have a higher intrinsic thrombotic risk when in AF, would be easily managed in rhythm because of thrombotic score usually lower than the bleeding scores.

2) Antiarrhythmic therapy. Chelation therapy is the mainstay of arrhythmia management and iron overload has to be managed before the administration of any antiarrhythmic drugs. Very few studies have evaluated the use of antiarrhythmic drugs for controlling AF in thalassemic patients. Regional guidelines as well as panels of international experts suggest that the treatment should not differ from the general population $[18,29,30]$. Considering that non negative-inotrope drugs should be preferred. Furthermore, between different antiarrhythmic drugs, those with frequent pro arrhythmic effects should be avoided [29], keeping in mind the possible drugs' interactions in these multiple pathologic patients. Finally, the frequent compresence of iron-induced endocrine disease, potentially causing arrhythmia or modifying the effect of specific drugs, must be considered when choosing the antiarrhythmic therapy [18,31]. Amiodarone is often successful in controlling atrial arrhythmias and can be a powerful temporizing measure during intensive iron chelation. Long-term therapy may be complicated by hypothyroidism because of the frequent association with iron-mediated thyroid injury. However, amiodarone therapy can often be successfully terminated after 6 to 12 months, as soon as successful chelation is obtained [18].

3) Anticoagulant therapy. Atrial fibrillation in $\beta$-TM is associated with an increased risk for stroke that may be exacerbated by asplenia [32,33]. Anticoagulation is generally recommended when heart failure or a history of stroke coexists but a careful risk/benefit ratio assessment 
should be undertaken before anticoagulation is started [34]. Liver function, platelet count, and renal function assessments may help establish the bleeding risk and should integrate the wellknown CHADSVASc and HASBLED scores [35]. Due to the documented prothrombotic tendencies, permanent anticoagulation has to be considered at an early stage in all patients with the only exception of the mildly affected group, who experience infrequent, short-lasting bouts of AF [18]. Warfarin is the most used anticoagulant. Only one study explored the effectiveness of the new oral anticoagulant rivaroxaban in a small group of patients with $\beta$-TM and AF, and none of the patients experienced any thrombotic or bleeding event during follow-up [36]. Again, drug interactions should be carefully taken into consideration.

4) Catheter ablation therapy. For those patients who cannot tolerate antiarrhythmic drugs and those whose AF is poorly controlled, despite the use of such medication, catheter ablation (CA) should be considered. It has been shown to be a safe and effective alternative to antiarrhythmia in both paroxysmal and persistent AF [37-39]. Current AF guidelines recommend ablation for patients with symptomatic AF, who have failed an antiarrhythmic drug therapy based on evidence (class I), but acknowledge that ablation may be used in some patients before a trial of antiarrhythmic drugs (class IIa) [35]. Catheter ablation indications for $\beta$-TM do not differ from those for the general population, but as experts suggest it should be reserved for patients who have undergone successful removal of cardiac iron documented with CMR [18]. Considering the increasing prevalence of $\mathrm{AF}$ in $\beta$-TM patients and the challenges with antiarrhythmic pharmacological therapy, CA is expected to have a greater role in the future. Currently, success rates for AF ablation in non thalassemic patients are quoted as being between 70.0 and $80.0 \%$ for the eradication of the arrhythmia, albeit with a 10.0 to $15.0 \%$ likelihood of requiring more than one procedures [40]. There are no studies at the moment evaluating the success rate of catheter ablation on $\beta$-TM patients. Our experience, as well as discussion with experts at other centers, seems to agree that even young patients with thalassemia and previous history of iron overload, would have a higher level of electrical remodeling with patchy distribution of low voltages zones and diffused fragmented potentials that pave the way to complex atypical flutter. The pathophysiology of supraventricular tachycardia and AF in the $\beta$-TM population may, in fact, be different, so that equivalent success rates should not be assumed to apply to this special group of patients. Given the already compromised substrate, with almost always dilated atria and the effect of exposure of myocardial atrial cells to iron toxicity, it is sometimes more than a feeling that such patients should be referred early to CA to make the most of this therapeutic opportunity.

\section{Conclusions}

The aging of the $\beta$-TM population, mostly due to improved chelation therapy, is leading to morbidities that are new to this group of patients or used to be less frequent in this specific group, such as AF. Atrial fibrillation is a growing problem for $\beta$-TM patients. Iron chelation is the first and essential therapeutic strategy; as regards more specific therapeutic options including antiarrhythmic treatment, anticoagulant therapy, and CA, $\beta$-TM patients' management does not differ from the general population. Nevertheless, little is known about the specificity of $\beta$-TM iron overload-induced cardiomyopathy and its potential specific therapeutic implication. Careful selection of patients with higher risk of developing AF to address to cardiac monitoring is mandatory in order to early detect its onset and to evaluate the opportunity of prophylactic 
anticoagulation treatment and rhythm management strategies. Based on the published literature in the field, we propose an active screening of all thalassemic patients including personal history of iron cardiac overload, thyroid disease and symptoms such as palpitation or effort dyspnea and regular check of 12-lead electrocardiograms (ECGs), 24-Holter ECG and echocardiographic characteristics that have been related to the onset of AF. If a patient has a high number of these risk factors, intensive follow-up with external or internal loop recorder might be considered. Once an AF diagnosis has been made, a concern due to anticoagulation therapy as the intrinsic higher thrombotic risk of this disease should be considered when calculating risk scores that have been extrapolated from non thalassemic populations. Rate control strategies should be pursued taking into account the potential for heart failure, while rhythm control strategies should rely first on optimal iron chelation, short periods of antiarrhythmic drugs and early referral to ablation procedure to avoid complex electrical remodeling of the atria.

\section{Disclosure statement}

The authors report no conflicts of interest. The authors alone are responsible for the content and writing of this article.

\section{References}

[1] Galanello R, Origa R. $\beta$-Thalassemia. Orphanet J Rare Dis. 2010;5:11.

[2] Olivieri NF, The $\beta$-thalassemias. N Engl J Med. 1999;341(2):99-109.

[3] Barbero U, Destefanis P. An Indian-look right into restrictive cardiomyopathies. Indian Heart J. 2015;67(6):512-513.

[4] Borgna-Pignatti C, Rugolotto S, De Stefano P, et al. Survival and disease complications in thalassemia major Ann NY Acad Sci. 1998;850:227-231.

[5] Thalassaemia International Federation guidelines for the clinical management of thalassemia. Nicosia (Cyprus): Thalassaemia International Federation, 2008. ISBN-13: 9789962-623-70-9.

[6] Flint J, Harding RM, Boyce AJ, Clegg JB. The population genetics of the haemoglobinopathies. Bailliére’s Clin Haematol. 1998;11(1):1-51.

[7] Kostopoulou AG, Tsiapras DP, Chaidaroglou AS, et al. The pathophysiological relationship and clinical significance of left atrial function and left ventricular diastolic dysfunction in $\beta$-thalassemia major. Am J Hematol. 2014;89(1):13-18.

[8] Kremastinos DT, Farmakis D. Iron overload cardiomyopathy in clinical practice. Circulation. 2011;124(20):2253-2263

[9] Barbero U, Destefanis P, Pozzi R, et al. Exercise stress echocardiography with tissue Doppler Imaging (TDI) detects early systolic dysfunction in $\beta$-thalassemia major patients without cardiac iron overload. Mediterr J Hematol Infect Dis. 2012;4(1):e2012037.

[10] Barbero U, Longo F Destefanis P, et al. Worsening of myocardial performance index in $\beta$-thalassemia patients despite permanently normal iron load at MRI: a simple and cheap index reflecting cardiovascular involvement? IJC Metabolic \& Endocrine. 2016;13(?):41-44.

[11] Buja LM, Roberts WC. Iron in the heart: etiology and clinical significance. Am J Med. 1971;51(2):209-221.

[12] Russo V, Rago A, Pannone B, et al. Atrial fibrillation and $\beta$ thalassemia major: the predictive role of the 12-lead electrocardiogram analysis. Indian Pacing Electrophysiol J. 
2014;14(3):121-132.

[13] Rago A, Russo V, Papa AA, et al. The role of the atrial electromechanical delay in predicting atrial fibrillation in $\beta$-thalassemia major patients. J Interv Card Electrophysiol. 2017;48(2):147-157.

[14] Bell RM, Mohamed S, Ako EA, et al. The prevalence and risk factors for atrial fibrillation in $\beta$-thalassemia major: a cross-sectional study in a UK specialist cardio-haematology clinic. ESC Congress, 2015; Poster session 6: Atrial fibrillation VI. Eur H Jour. 2015;36(1):916. [15] Coumel P. Cardiac arrhythmias and the autonomic nervous system. J Cardiovasc Electrophysiol. 1993;4(3):338-355.

[16] Kuryshev YA, Brittenham GM, Fujioka H, et al. Decreased sodium and increased transient outward potassium currents in iron-loaded cardiac myocytes: implications for the arrhythmogenesis of human siderotic heart disease. Circulation. 1999;100(6):675-683.

[17] Kirk P, Roughton M, Porter JB, et al. Cardiac T2* magnetic resonance for prediction of cardiac complications in thalassemia major. Circulation. 2009;120(20):1961-1968.

[18] Pennell DJ, Udelson JE, Arai AE, et al.; American Heart Association Committee on Heart Failure and Transplantation of the Council on Clinical Cardiology and Council on Cardiovascular Radiology and Imaging. Cardiovascular function and treatment in $\beta$-thalassemia major: a consensus statement from the American Heart Association. Circulation.

2013;128(3):281-308.

[19] Ozdogan O, Alp A, Turker M, Atabey B. Determination of early cardiac deterioration in $\beta$-thalassaemia major by echocardiography. Acta Cardiol. 2013;68(3):299-305.

[20] Russo V, Rago A, Pannone B, et al. Early electrocardiographic evaluation of atrial fibrillation risk in $\beta$-thalassemia major patients. Int J Hematol. 2011;93(4):446-451.

[21] Aessopos A, Farmakis D, Karagiorga M, et al. Cardiac involvement in thalassemia intermedia: a multicenter study. Blood. 2001;97(11):3411-3416.

[22] Franzoni F, Galeta F, Di Muro C, et al. Heart rate variability and ventricular late potentials in $\beta$ thalassemia major. Haematologica. 2004;89(2):233-234.

[23] Gurses D, Ulger Z, Levent E, et al. Time domain heart rate variability analysis in patients with thalassaemia major. Acta Cardiologica. 2005;60(5):477-482.

[24] Amoozgar H, Zeighami S, Haghpanah S, Karimi M. A comparison of heart function and arrhythmia in clinically asymptomatic patients with $\beta$ thalassemia intermedia and $\beta$ thalassemia major. Hematology. 2017;22(1):25-29.

[25] Yetimakman AF, Oztarhan K, Aydogan G. Comparison of tissue Doppler imaging with MRI t2* and 24-hour rhythm holter heart rate variability for diagnosing early cardiac impairment in thalassemia major patients. Pediatr Hematol Oncol. 2014;31(7):597-606.

[26] Kolios M, Korantzopoulos P, Vlahos AP, et al. Electrocardiographic abnormalities and arrhythmic risk markers in adult patients with $\beta$ thalassemia major. Int J Cardiol.

2016;15(221):932-936.

[27] Prystowsky EN, Padanilam BJ, Fogel RI. Treatment of atrial fibrillation. JAMA. 2015;314(3):278-288.

[28] Russo V, Papa AA, Rago A, Nigro G. Atrial fibrillation in $\beta$ thalassemia major: how to perform effective screening and early detection. Hematology. 2017;22(6):368-369.

[29] ADTL, Regione Lombardia. Linee guida per la prevenzione e il trattamento delle complicanze della talassemia. 1999;1(1):1-21.

[30] Fondazione L. Giambrone, Linee guida per le complicanze cardiache nella talassemia major. Primo aggiornamento. 2008;1(1):7-24. 
[31] Brittenham GM. Iron-chelating therapy for transfusional iron overload. N Engl J Med. 2011;364(2):146-156.

[32] Vassilopoulou S, Anagnostou E, Paraskevas G, Spengos K. Etiology and treatment of ischaemic stroke in patients with $\beta$-thalassemia major. Eur J Neurol. 2011;18(12):1426-1428. [33] Taher A, Mehio G, Isma'eel H, Cappellini MD. Stroke in thalassemia: a dilemma. Am J Hematol. 2008;83(4):343.

[34] Auger D, Pennell DJ. Cardiac complications in thalassemia major. Ann NY Acad Sci. 2016;1368(1):56-64.

[35] Kirchhof P, Benussi S, Kotecha D, et al. 2016 ESC Guidelines for the management of atrial fibrillation developed in collaboration with EACTS. Europace. 2016;18(11):1609-1678.

[36] Apostolou C, Klonizakis P, Mainou M, et al. Rivaroxaban use in patients with hemoglobinopathies. Hemoglobin. 2017;41(3):223-224.

[37] Wynn GJ, Das M, Bonnett LJ, et al. Efficacy of catheter ablation for persistent atrial fibrillation: a systematic review and meta-analysis of evidence from randomized and nonrandomized controlled trials. Circ Arrhythm Electrophysiol. 2014;7(5):841-852.

[38] Takahashi Y, Yamashita S, Suzuki M, et al. Efficacy of catheter ablation of focal sources in persistent atrial fibrillation. J Cardiovasc Electrophysiol. 2018;29(4):559-565.

[39] Anselmino M, Ferraris F, Cerrato N, et al. Left persistent superior vena cava and paroxysmal atrial fibrillation: the role of selective radio-frequency transcatheter ablation. J Cardiovasc Med (Hagerstown). 2014;15(8):647-652.

[40] Gaita F, Scaglione M, Battaglia A, et al. Very long-term outcome following transcatheter ablation of atrial fibrillation. Are results maintained after 10 years of follow up? Europace. 2018;20(3):443-450. 